Research Article

\title{
Optimization of the UWB Feed Antenna Position in Reflector Applications
}

\author{
Nurhan Türker Tokan \\ Department of Electronics and Communications Engineering, Yildiz Technical University, Esenler, 34220 Istanbul, Turkey \\ Correspondence should be addressed to Nurhan Türker Tokan; nurhanturker@gmail.com
}

Received 21 October 2013; Accepted 11 February 2014; Published 17 March 2014

Academic Editor: Ahmed A. Kishk

Copyright (C) 2014 Nurhan Türker Tokan. This is an open access article distributed under the Creative Commons Attribution License, which permits unrestricted use, distribution, and reproduction in any medium, provided the original work is properly cited.

In reflector system design, achieving high stability of phase center position with changes in frequency in reflector feed antennas is highly desired. However, obtaining highly stable phase center is not possible for UWB feed antennas, specially for planar ones. Thus, an optimum positioning for the UWB feed antenna should be defined. Optimization of the positioning of the feed antenna is essential since this process lowers resulting phase error losses significantly. In this work, a novel method for optimizing the UWB feed position of a prime focus reflector antenna from phase and amplitude recordings of the measured radiated field is introduced. An automatic and fast design procedure, based on Genetic Algorithms, is described. The proposed methodology has been numerically and experimentally assessed. The procedure is introduced by an application example to one of the most commonly used UWB feed antennas in high-performance reflector antenna systems: Linear Tapered Slot Antenna (LTSA). A LTSA antenna operating in $6-25 \mathrm{GHz}$ frequency band is designed and manufactured. The performance of the method is quantified in terms of its phase error losses in $E$ - and $H$-planes for reflector illumination.

\section{Introduction}

One of the critical issues in reflector antenna system design is obtaining a stable phase center at the feed antenna and preventing efficiency reduction due to the defocusing at the frequency band extremes [1]. If it was possible to build a feed antenna with a unique phase center and place it to the focus of a perfect paraboloidal reflector, it would be possible to eliminate phase error losses $[2,3]$. However, this is possible only at narrow band systems. Since there is an increasing interest in extremely large bandwidth high-performance applications, the usage of ultrawide band (UWB) antennas as reflector feeds is unavoidable. In any wide band application, phase error losses due to the variation in phase center location with frequency are expected. This is because the antenna phase center will be coincident with the focus of the reflector only at one particular frequency and when displaced at other frequencies phase error losses will increase. To determine the phase center location of a radiating element, spherical measurements of the antenna are mostly used [4]. The phase center is determined experimentally by finding the equiphase sphere in the radiation direction of the antenna. The center of the surface corresponds to the phase center of the antenna under test. In literature, phase center variations of general types of antennas are investigated. These include planar loop antenna, coupled planar dipole antenna, horn antenna, radial line helical array antenna, small feeds, and Vivaldi antennas [5-12]. The amount of the resulting phase error loss in a reflector antenna system is related to the defocusing of the phase center from reflector focus. Long feeding elements exhibit more losses since the variation of the phase center location between lowest and highest frequency would be more. In a high-performance reflector system, long feeding elements are required to achieve a sufficiently high directivity. For such long elements, high phase center instability causes considerable phase error loss (PEL) due to axial defocusing and astigmatism in the reflector system. To minimize phase error losses of the reflector system, an appropriate position to locate the feed antenna should be determined. If the feed antenna was a narrow band antenna, it would be sufficient to place it to the focus of the reflector antenna. However, for an UWB antenna, phase center and corresponding PELs 
are different at every frequency. One way to reduce the PELs due to UWB feed antenna may be to design the UWB feed antenna with an appropriate profile giving a stable phase center. This procedure is applied to horn antennas in [1] and high stability of the phase center position with respect to frequency is achieved at the frequency band extremes. However, this process is not applicable to planar UWB antennas. Thus, a suitable positioning of the feed antenna should be defined by accounting the consequential PELs at every frequency.

In [13], the necessity of an optimization for the positioning of the feed antenna in reflector applications is emphasized. The goal of this contribution is to propose an optimization technique for the determination of the feed antenna positioning giving the lowest PELs within the predetermined frequency band. In this approach, radiation patterns of the feed antenna are used. The feed patterns at each frequency are measured and inputted to the optimization tool. The position giving the lowest total PEL for the reflector system is determined by shifting the feed antenna pattern on $z$ direction at every frequency and recalculating the resulting PEL at every iteration. This design procedure is automated with Genetic Algorithm (GA) approach. GA is preferred due to its user friendly usage and fast convergence capability [14]. This simple and useful approach can be applied by using any optimization technique.

As an application example of this approach, the optimization process is applied to a directive, UWB antenna: Linear Tapered Slot Antenna (LTSA). It is a well-known, widely used, wideband and compact slot antenna [15] and is proved to be an ideal candidate for reflector feed applications in [13]. It has a linear or piecewise-linear taper instead of the exponential curved one of the well-known Vivaldi antenna [16]. Their characteristics are similar to each other, particularly when they have a similar opening angle. They are travelling wave type antennas with a directional radiation along its aperture. This antenna type is widely used as a feed for the parabolic dish reflector and is the strongest candidate for ultrawide band (UWB) high-performance applications (e.g., Square Kilometer Array radio telescope) [17]. With this work, the ideal positioning of the LTSA feed antenna operating in the 4.16:1 frequency band is determined by combining the far field radiation pattern of the LTSA with GA. Its phase center locations in $E$ - and $H$-planes are found within the operation band and the losses due to axial defocusing are investigated. Some application examples of the reflector system are given to demonstrate the approach. The paper is structured as follows. In the next section, the concept of phase center variation is defined. In Section 3, optimization procedure of the feed antenna with GA is defined with given examples of LTSA. The considerations on the results are given in the last section.

\section{PELs in UWB Feed Antenna}

In practice, the phase center of an antenna can be defined as the point on the feed that leads to minimum phase error loss [2]. At the phase center, the electromagnetic radiation spreads spherically outward, with the phase of the signal being equal

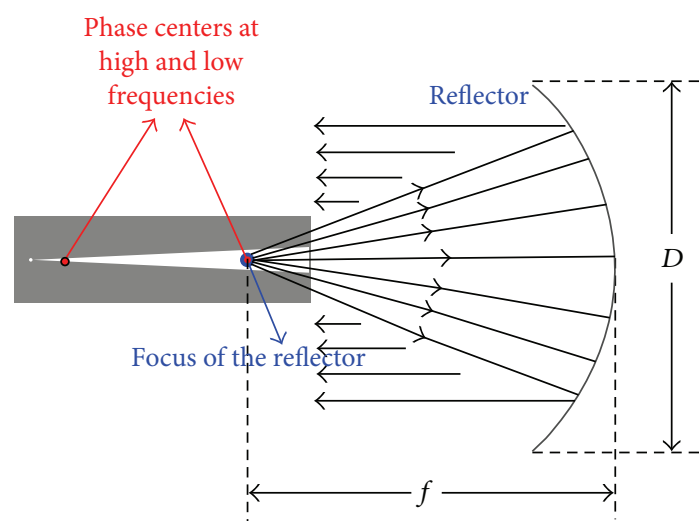

FIgURE 1: Phase centers of an UWB antenna at high and low frequencies.

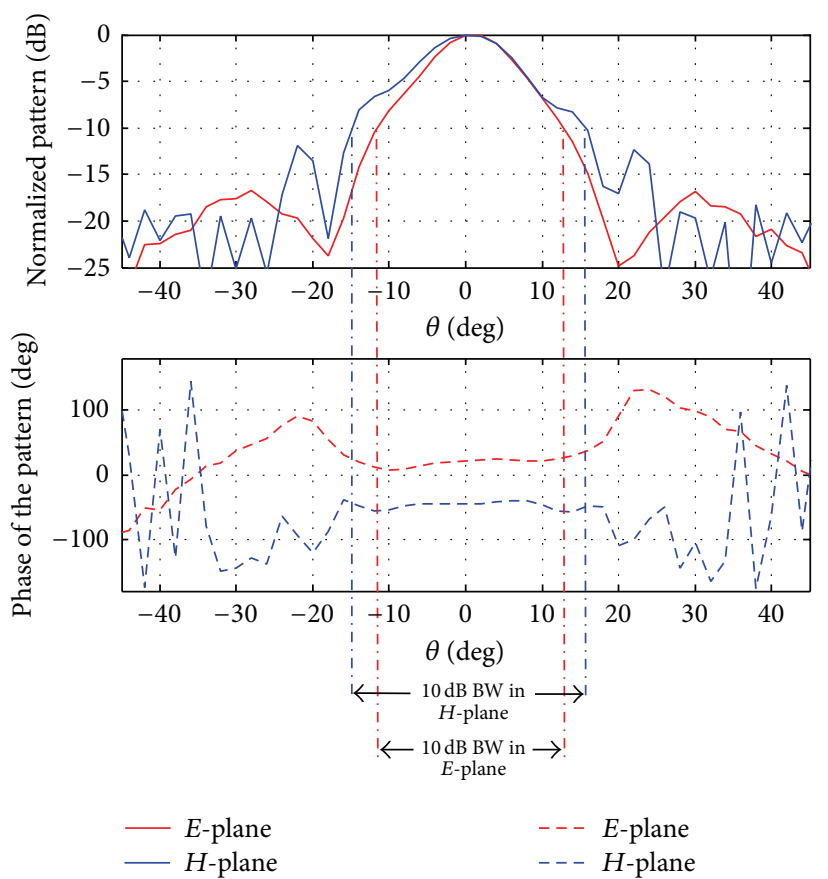

FIGURE 2: Radiation patterns and corresponding phase variations at phase centers in $E$ - and $H$-planes.

at any point on the sphere. Paraboloidal reflector transforms the spherical wave radiated by the feed into a plane wave. The phase center locations at low and high frequencies would be different from each other. In Figure 1, the phase center locations of a planar UWB antenna are demonstrated. The red dots are the phase center positions at the highest and lowest frequencies. The blue circle is the focus point of the paraboloidal reflector. With its current positioning, it would be possible to use the reflector system perfectly at low frequencies of the band. However, at high frequencies there will be distance between the phase center location and the focus point of the reflector. This will result as phase error losses in the system.

Due to the variation in phase center location with frequency as demonstrated in Figure 1, the feed phase center 


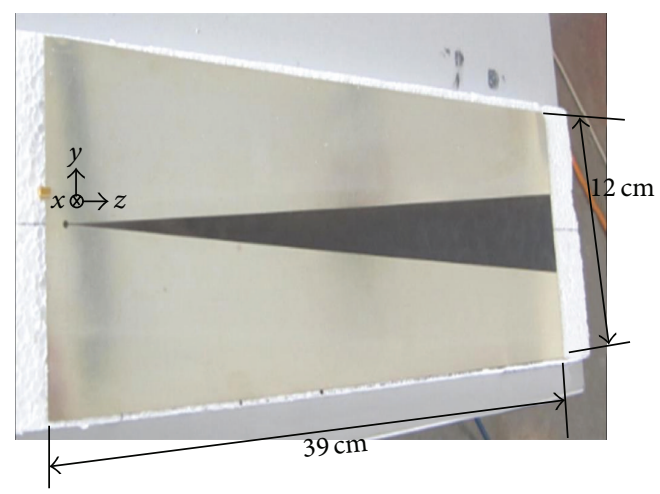

Figure 3: UWB Linear Tapered Slot Antenna.

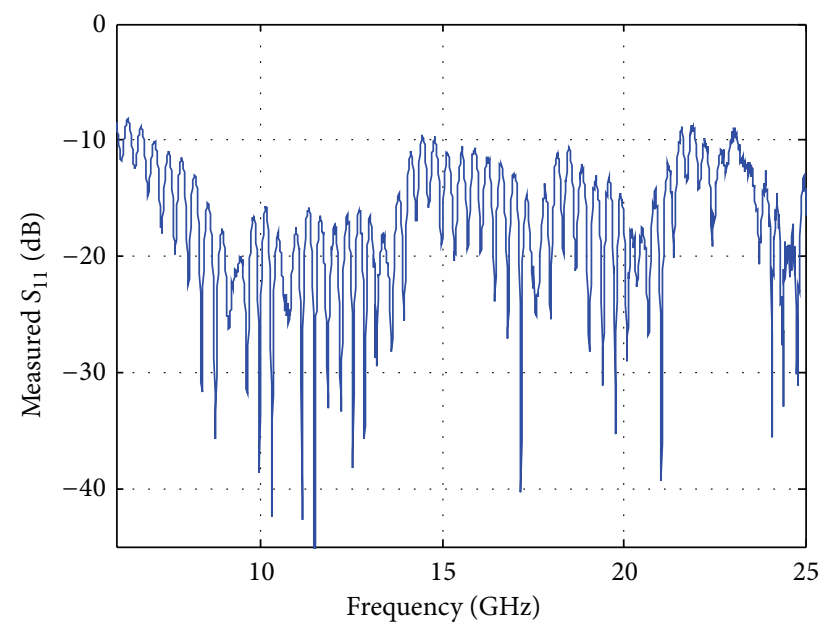

FIGURE 4: Measured return loss of the LTSA.

cannot be placed at the focus of the reflector in UWB applications and this results as axial defocusing. We can estimate the phase error loss (PEL) due to axial defocusing by integrating the feed pattern:

$$
\mathrm{PEL}=\frac{\left|\int_{0}^{2 \pi} \int_{\psi_{b}}^{\psi_{o}} E(\psi, \varphi) \tan (\psi / 2) d \psi d \varphi\right|^{2}}{\left[\int_{0}^{2 \pi} \int_{\psi_{b}}^{\psi_{o}}|E(\psi, \varphi)| \tan (\psi / 2) d \psi d \varphi\right]^{2}}
$$

where $E(\psi, \varphi)$ is the feed pattern and $\psi_{b}=2 \tan ^{-1}[b /(2 f)]$. Here $b$ is the central blockage radius of the feed antenna. The half subtended angle of the reflector, $\psi_{o}$, is related to $f / D$ by

$$
\psi_{o}=2 \tan ^{-1} \frac{1}{4 f / D}
$$

At the phase center, the phase value of the measured pattern should be constant in a certain angular area of interest. The efficiency of the reflector antenna peaks when the $10 \mathrm{~dB}$ beamwidth of the feed antenna is approximately equal to the subtended angle of the reflector. Thus, in the determination of the phase center locations, $10 \mathrm{~dB}$ beamwidth of the feed antenna in $E$ - and $H$-planes for the given frequency is considered. Phase center locations of an UWB antenna can

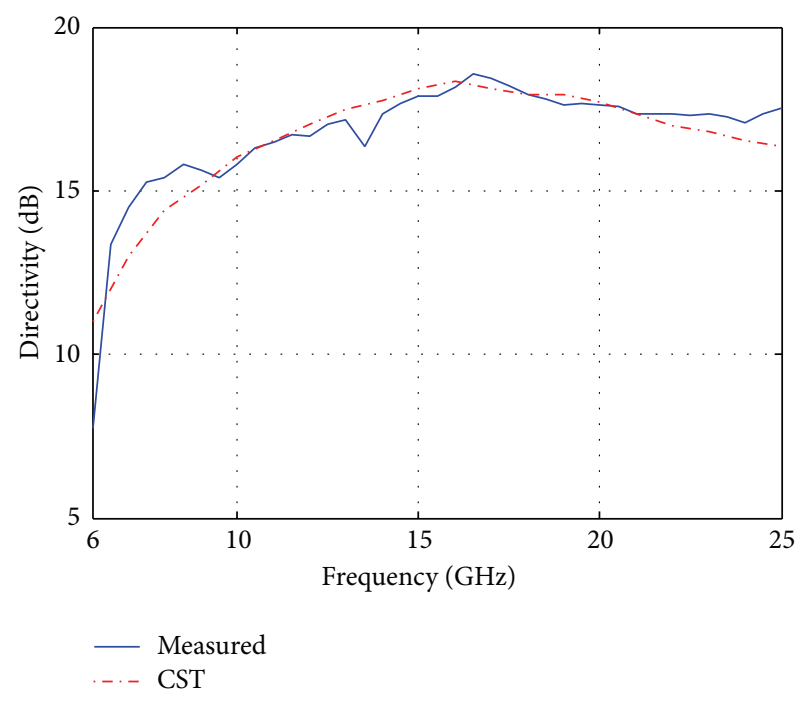

FIGURE 5: Measured and simulated directivities of the LTSA.

be obtained from simulation or measurement results. In this work, it is assumed as the location where almost constant phase within $10 \mathrm{~dB}$ beamwidth is obtained at the phase of the measured pattern. This is demonstrated in Figure 2 for a planar UWB antenna at $12 \mathrm{GHz} .10 \mathrm{~dB}$ beamwidth values are determined from the feed pattern and position giving the lowest phase variation at that frequency is expected to be the phase center position of that particular frequency.

Unequal phase center locations in $E$ - and $H$-planes introduce phase error losses due to astigmatism. It is detected by the depth of the nulls in the $E$ - and $H$-planes. Phase error loss due to astigmatism is not as severe as the losses due to axial defocusing [2].

\section{Optimization of the Feed Antenna Position}

GA optimizers are well-known tools in the electromagnetic community. They are particularly effective when the goal is to find an approximate global optimum (maximum or minimum) for multimodal functions [14]. Experimental data, numerically generated data, or analytical functions may be employed in a wide variety of optimization problems. An overview of GAs for electromagnetic optimization problems can be found in $[18,19]$.

In this section, an automatic design procedure for reflector systems based on GA is demonstrated. GA is used to determine the positioning of the UWB feed antenna of the reflector antenna. The procedure is simple. First a feed antenna is designed and its far field patterns are measured. The patterns are inputted to the GA. The total PELs for Eand $H$-planes within the band of interest are investigated. The best solution in the frequency band is obtained based on the predetermined constraints. The procedure is introduced with given examples of a reflector system with Linear Tapered Slot Antenna feed working in the $6-25 \mathrm{GHz}$ frequency band. The position of the feed antenna is optimized to give minimum total phase error loss in the defined band/subbands. 


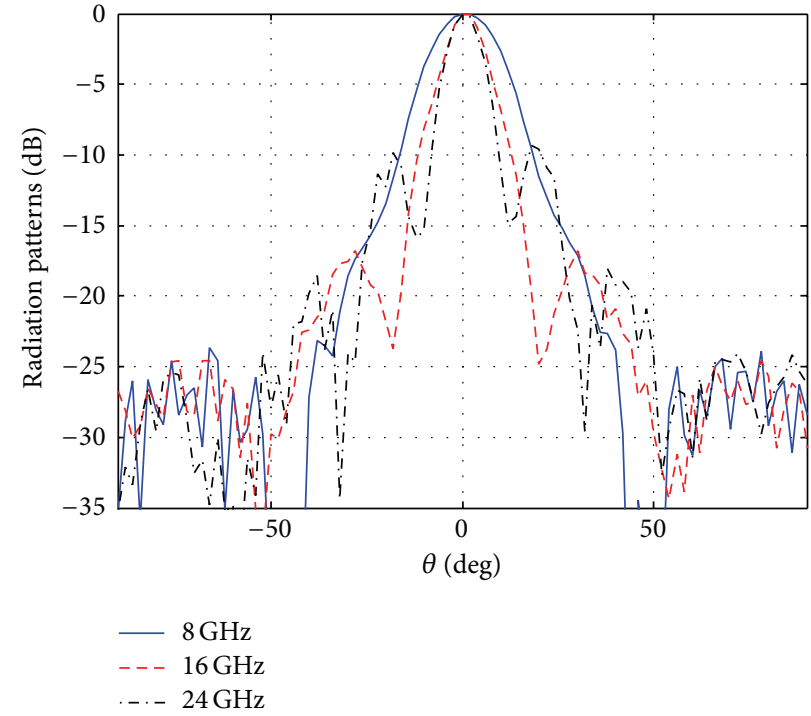

(a)

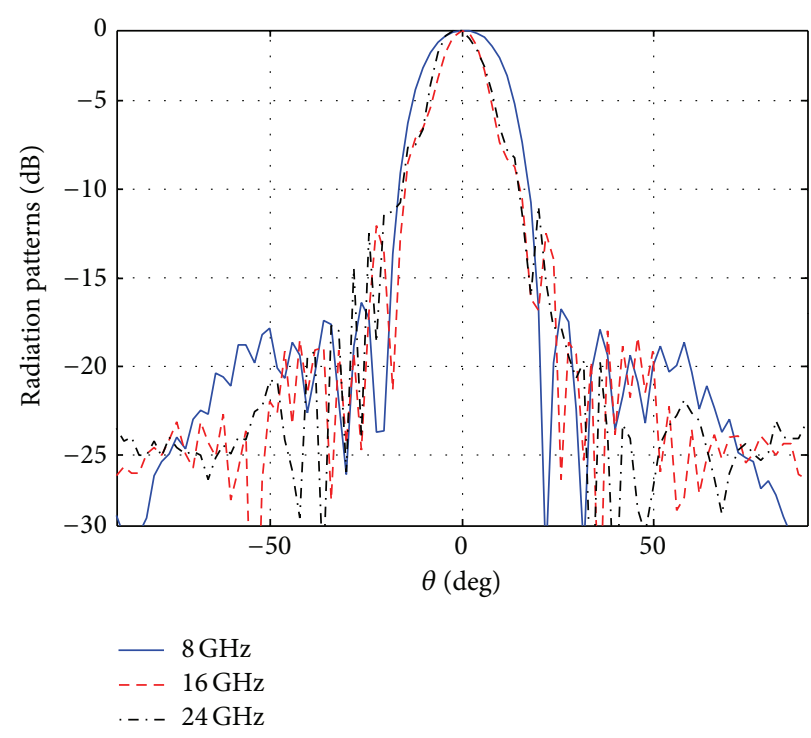

(b)

FIgURE 6: Measured copolarized radiation patterns of LTSA: (a) E-plane; (b) $H$-plane.

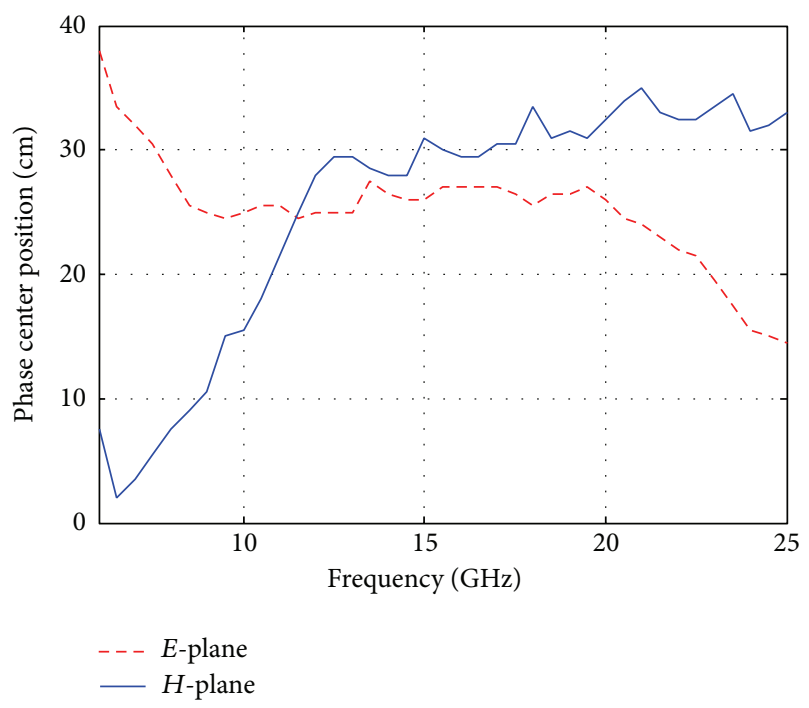

Figure 7: Variation of the phase center positions with frequency at $E$ - and $H$-planes.

3.1. Linear Tapered Slot Antenna (LTSA). LTSA is an end fire radiator usually supported on a thin, low $\varepsilon_{r}$ substrate. Despite the completely planar geometry of LTSA, it can produce almost symmetric radiation patterns in the $E$ - and $H$-planes $[13,15]$. As the length of the antenna increases, its beam width narrows and the directivity increases. Thus, to obtain a high directivity, a long LTSA is designed as given in Figure 3 with its dimensions. The antenna is designed to operate in the band of $6 \mathrm{GHz}-25 \mathrm{GHz}$ (4.16:1 bandwidth). The length of the tapering is approximately seven wavelengths at the lowest frequency and its flaring is $6^{\circ}$. The dielectric constant of the considered material is chosen to be $\varepsilon_{r}=2.2$ with thickness of $t=0.9 \mathrm{~mm}$.
3.2. Measurement Results. LTSA has been measured using an Agilent vector network analyzer, which is able to perform measurements up to $70 \mathrm{GHz}$. The reflection behaviour of the LTSA has been investigated in terms of $S_{11}$ and is given by Figure 4 . The transmit-receive antenna link has been characterized in terms of $S_{12}$ with the measurement being set up where the antennas were placed at about $1.6 \mathrm{~m}$.

The feed antenna has been also analyzed by means of the commercial code CST [20] based on the FIT method. Simulated and measured directivities of LTSA as the function of the frequency are given in Figure 5.

The experimental normalized far field radiation patterns of the feed antenna are given for $E$ - and $H$-planes at 8,16 , and $24 \mathrm{GHz}$ in Figures 6(a) and 6(b), respectively. Radiation patterns are used in the integration equation given by (1). The $10 \mathrm{~dB}$ beamwidth in $H$-plane varies between $45^{\circ}$ and $30^{\circ}$ between $6 \mathrm{GHz}$ and $25 \mathrm{GHz}$. Similarly, it varies between $45^{\circ}$ and $20^{\circ}$ in E-plane. Thus, the feed subtended angle of the reflector is assumed to be $40^{\circ}$. This results in the focal length to reflector diameter ratio $(f / D)$ of approximately 1.4 for the reflector system. The pattern at each frequency is shifted in $z$ direction and the PELs are optimized to give the lowest phase error loss.

In Figure 7, the phase center variations of the LTSA are given at $E$ - and $H$-planes with respect to frequency. In $E$ plane, phase center location is at $z=38 \mathrm{~cm}$ at the lowest frequency while it is at $z=14.5 \mathrm{~cm}$ at the highest frequency. It is at $z=8 \mathrm{~cm}$ and $z=32.5 \mathrm{~cm}$ in $H$-plane at the lowest and highest frequencies, respectively. Here, $z$ is the axis along the tapering of the feed antenna as given in Figure 3. The reference point for $z$ is the end of the slot line and beginning of the flaring. The variation between the lowest and highest frequencies in $H$-plane is $24.5 \mathrm{~cm}$ which is about 20 wavelengths at the highest frequency. It is $23.5 \mathrm{~cm}$ in $E$ plane which is also equal to almost 20 wavelengths at $25 \mathrm{GHz}$. 


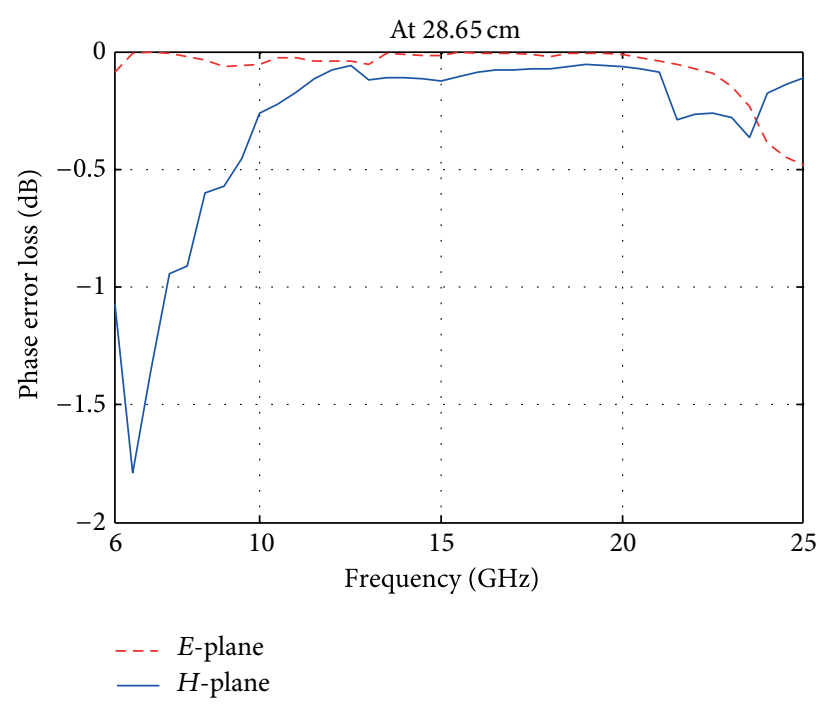

(a)

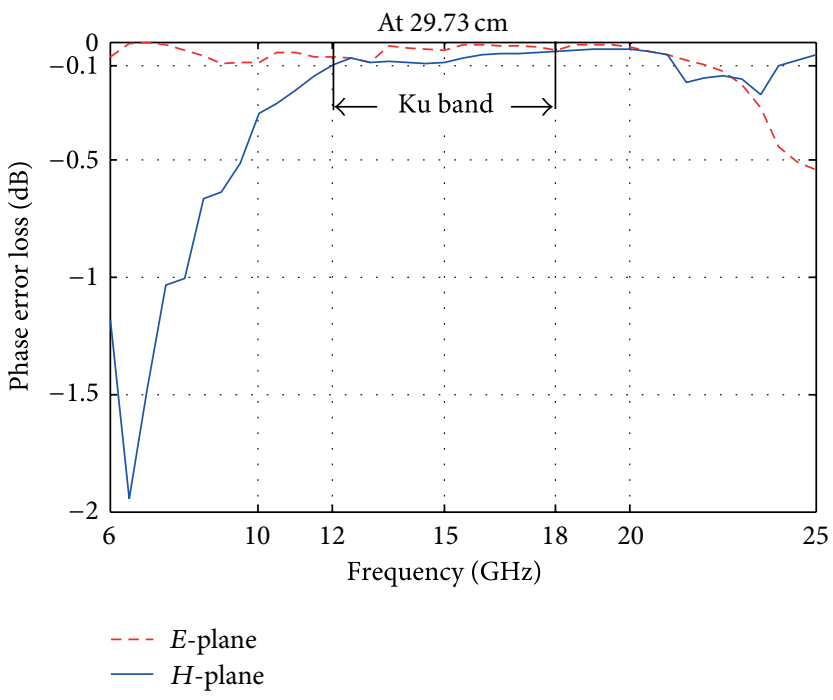

(b)

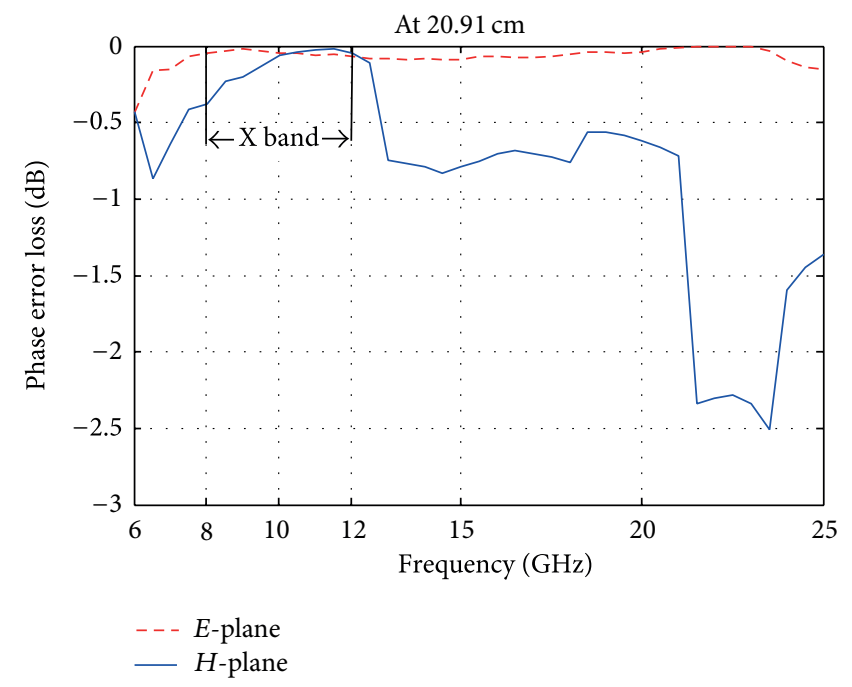

(c)

FIgURE 8: PEL variations after optimization. (a) Whole frequency band (6-25 GHz); (b) Ku band (12-18 GHz); (c) X band (8-12 GHz).

This distance between phase center locations at lowest and highest frequencies will cause the phase error losses due to axial defocusing.

3.3. Resulting Phase Error Losses. Genetic Algorithm Toolbox in MATLAB is used for the optimization process. Fitness function is defined by the integration of the feed pattern given by (1). GA finds the optimum positioning that gives the minimum total PEL within the defined frequency band. Optimization of the feed antenna positioning is essential since it may result in high losses in the reflector system. As an example, if LTSA was mounted to the focus of the reflector from the starting of its tapering $(z=0)$, the losses in $H$ plane would be at $10 \mathrm{~dB}$ levels and in $E$-plane it would be at $2 \mathrm{~dB}$ levels. In Figure 8(a), the corresponding losses when the feed antenna position is optimized with the aim of giving the lowest total PEL in the whole frequency band are given. Here, the best positioning of the feed antenna is determined as $z=28.65 \mathrm{~cm}$ if the full band of the reflector system would be used. The PEL level is less than $1.8 \mathrm{~dB}$ in the whole frequency band at $H$-plane and less than $0.5 \mathrm{~dB}$ at $E$-plane. It is clear that, with the current positioning of the feed antenna, the reflector may be used effectively in the middle frequencies of the band. At the edges of the band, PELs go higher. If the reflector system is aimed to be used at a subband of the whole frequency band, the optimization process should be performed according to these constraints. There are a number of radio frequency ranges in use in satellite communications such as $\mathrm{C}, \mathrm{X}, \mathrm{Ku}, \mathrm{Ka}$, and even EHG and $\mathrm{V}$ band. As an example, if the reflector system would be used in the Ku band, the optimization process should be repeated with frequency constraints and the resulting PEL variation would be as given in Figure 8(b). As can be observed, PELs for $E$ - and $H$-planes within the Ku band are all lower than $0.1 \mathrm{~dB}$. The lower side 


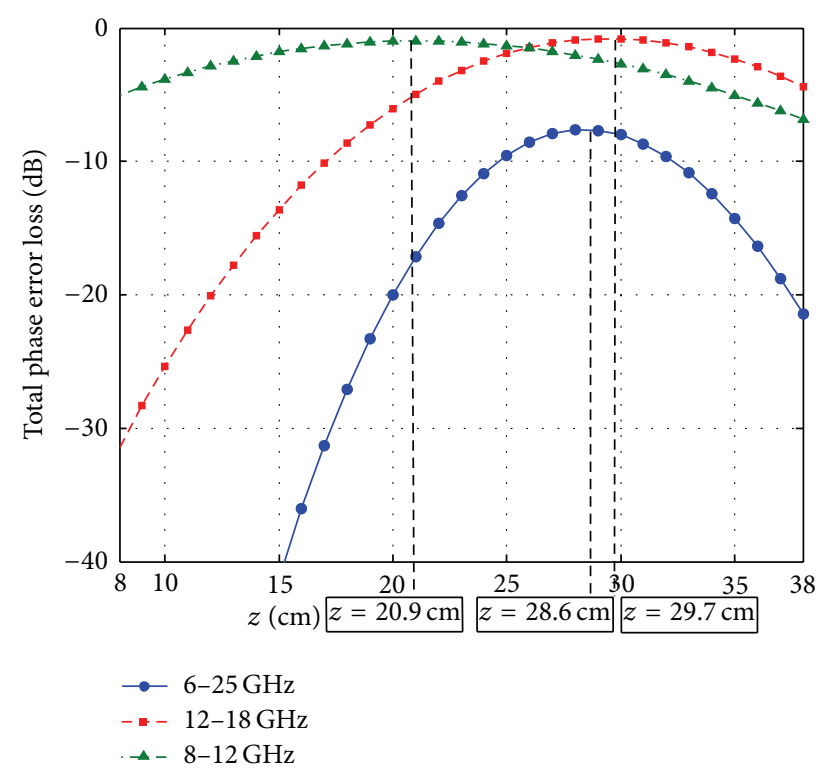

FIGURE 9: Total PEL variations with $z$ parameter.

of the frequency band is devoted to the X band. As observed from Figure 8(a), losses at that region of the band go below $1.5 \mathrm{~dB}$. If the optimization process was repeated for the $\mathrm{X}$ band, optimum positioning would be determined as $z=$ $20.91 \mathrm{~cm}$ and losses less than $0.4 \mathrm{~dB}$ at $H$-plane and $0.1 \mathrm{~dB}$ would be obtained as given in Figure 8(c).

Total PEL variation demonstration of the above given examples is given by Figure 9. Total phase error loss is the sum of the losses in $E$ - and $H$-planes. $1 \mathrm{GHz}$ frequency step is accounted. The values are calculated by $1 \mathrm{~cm}$ distances within the phase center variation bounds. The blue line refers to the variation in the 6-25 GHz band. Red and green line belong to the $\mathrm{Ku}$ and $\mathrm{X}$ band, respectively. It can be observed that the lowest total PEL is obtained at approximately the same values with GA results.

\section{Conclusion}

In reflector system design, a single feed that covers the entire frequency band of operation with a symmetric, directive pattern, dual linear polarization, and frequency invariant phase center and radiation pattern is desirable. For highly directive, UWB reflector systems, long feed antenna elements are used. These elements exhibit high phase center instability which results as phase error losses in reflector systems. Thus, an optimum positioning for the UWB feed antenna should be defined. Optimization of the positioning of the feed antenna is essential since this process lowers resulting phase error losses significantly.

In this work, a novel method for optimizing the UWB feed position of a prime focus reflector antenna from phase and amplitude recordings of the measured radiated field is introduced. The need for the optimization of the positioning for the planar UWB feed antennas in high-performance reflector applications is proved. An automatic and fast design procedure, based on Genetic Algorithms, is described. The procedure is introduced by an application example to one of the most commonly used UWB feed antennas in highperformance reflector antenna systems: Linear Tapered Slot Antenna. A prototype demonstrator has been manufactured and tested over a 4.16:1 bandwidth. The performance of the method is quantified in terms of phase error losses of LTSA in $E$ - and $H$-planes for reflector illumination.

\section{Conflict of Interests}

The author declares that there is no conflict of interests regarding the publication of this paper.

\section{References}

[1] L. Lucci, R. Nesti, G. Pelosi, and S. Selleri, "Phase centre optimization in profiled corrugated circular horns with parallel genetic algorithms," Progress in Electromagnetics Research, vol. 46, pp. 127-142, 2004.

[2] T. A. Milligan, Modern Antenna Design, Wiley-IEEE Press, 2005.

[3] C. A. Balanis, Antenna Theory Analysis and Design, WileyInterscience, 3rd edition, 2005.

[4] P. Li and L. Jiang, "The far field transformation for the antenna modeling based on spherical electric field measurements," Progress in Electromagnetics Research, vol. 123, pp. 243-261, 2012.

[5] Q. Wu, B. Jin, L. Bian, Y. Wu, and L. Li, "An approach to the determination of the phase center of Vivaldi-based UWB antenna," in Proceedings of the IEEE Antennas and Propagation Society International Symposium, pp. 563-566, Albuquerque, NM, USA, 2006.

[6] H. G. Schantz, "Dispersion and UWB antennas," in Proceedings of the International Workshop on Ultra Wideband Systems Joint with Conference on Ultra Wideband Systems and Technologies Joint (UWBST \& IWUWBS '04), pp. 161-165, Huntsville, Ala, USA, 2004.

[7] C. Zhao, "Analysis on the properties of a coupled planar dipole UWB antenna," IEEE Antennas and Wireless Propagation Letters, vol. 3, no. 1, pp. 317-320, 2004.

[8] H. Moheb, A. Sebak, and L. Shafai, "Phase centre analysis of array antennas and its significance for microwave landing system," in Proceedings of the 7th IEE International Conference on Antennas and Propagation (ICAP'91), pp. 213-216, York, UK, 1991.

[9] K. Z. Jadoon, S. Lambot, E. C. Slob, and H. Vereecken, "Analysis of horn antenna transfer functions and phase-center position for modeling off-ground GPR," IEEE Transactions on Geoscience and Remote Sensing, vol. 49, no. 5, pp. 1649-1662, 2011.

[10] L. Li, J. Zhang, and X. Li, "Theoretical calculation and simulation of phase center of a radial line helical array antenna," in Proceedings of the International Conference on Microwave and Millimeter Wave Technology (ICMMT '10), pp. 428-430, Chengdu, China, 2010.

[11] L. Shafai and A. A. Kishk, "Phase centre of small primary feeds and its effects on the feed performance," IEE Proceedings $H$ : Microwaves Optics and Antennas, vol. 132, no. 3, pp. 207-214, 1985. 
[12] N. T. Tokan, "Performance of Vivaldi antennas in reflector feed applications," Applied Computational Electromagnetics Society Journal, vol. 28, no. 9, pp. 802-808, 2013.

[13] N. T. Tokan, A. Neto, F. Tokan, and D. Cavallo, "Comparative study on pulse distortion and phase aberration of directive ultra-wideband antennas," IET Microwaves, Antennas \& Propagation, vol. 7, no. 12, pp. 1021-1026, 2013.

[14] S. L. Avila, W. P. Carpes Jr., and J. A. Vasconcelos, "Optimization of an offset reflector antenna using genetic algorithms," IEEE Transactions on Magnetics, vol. 40, no. 2, pp. 1256-1259, 2004.

[15] K. S. Yngvesson, T. L. Korzeniowski, Y. S. Kim, E. L. Kollberg, and J. F. Johansson, "The tapered slot antenna-a new integrated element for millimeter-wave applications," IEEE Transactions on Microwave Theory and Techniques, vol. 37, no. 2, pp. 365-374, 1989.

[16] P. J. Gibson, "The Vivaldi aerial," in Proceedings of the 9th European Microwave Conference, pp. 101-105, Brighton, UK, 1979.

[17] P. J. Hall, The Square Kilometer Array: An Engineering Perspective, Springer, 2005.

[18] R. L. Haupt, "An introduction to genetic algorithms for electromagnetics," IEEE Antennas and Propagation Magazine, vol. 37, no. 2, pp. 7-15, 1995.

[19] J. M. Johnson and Y. Rahmat-Samii, "Genetic algorithms in engineering electromagnetics," IEEE Antennas and Propagation Magazine, vol. 39, no. 4, pp. 7-21, 1997.

[20] The homepage of CST Microwave Studio, http://www.cst.com/. 

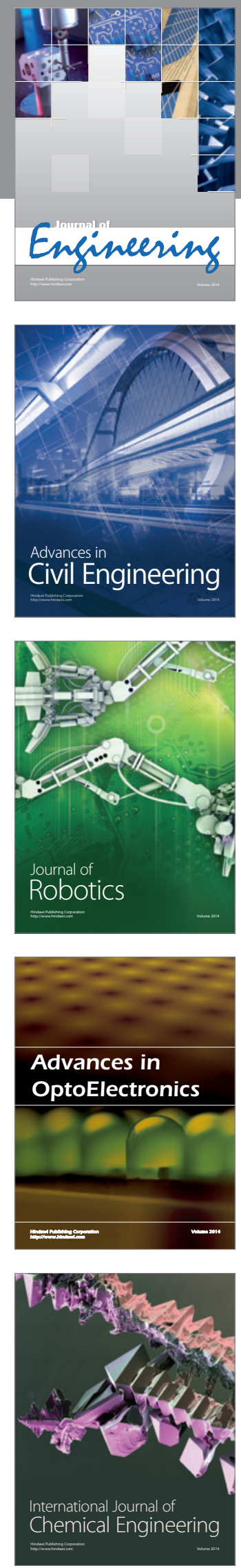

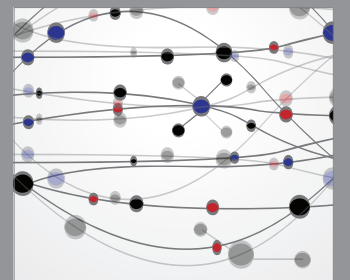

The Scientific World Journal
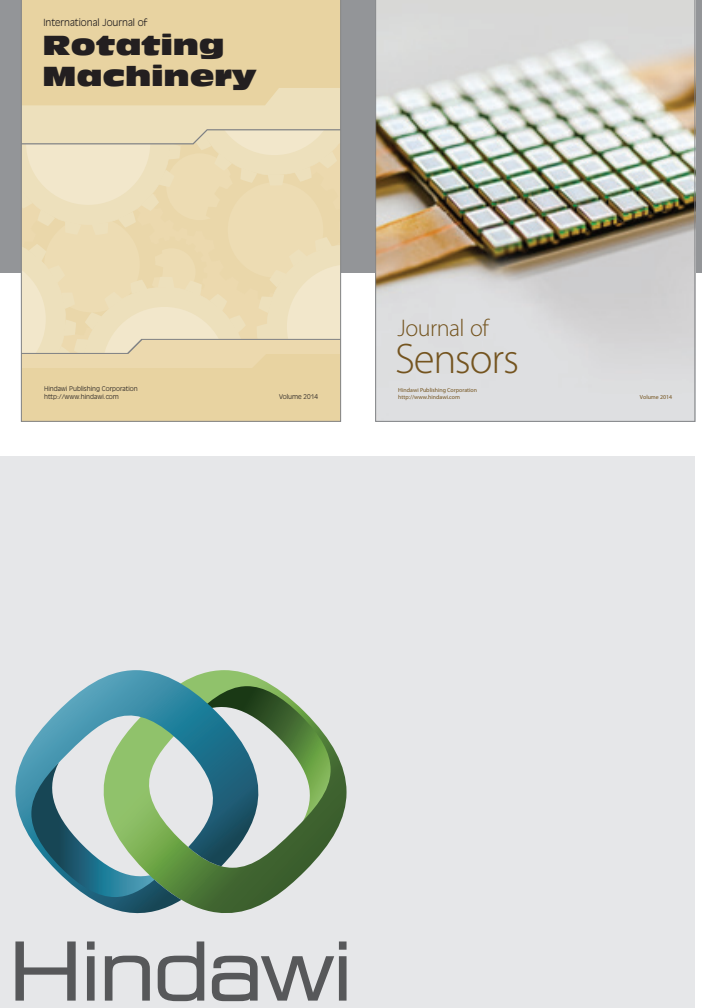

Submit your manuscripts at http://www.hindawi.com
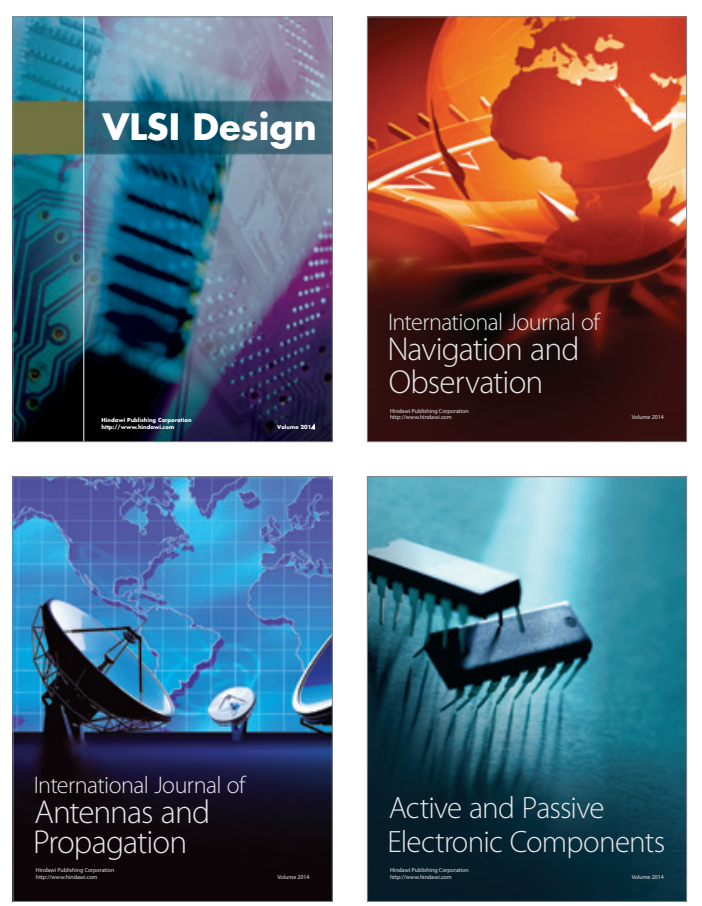
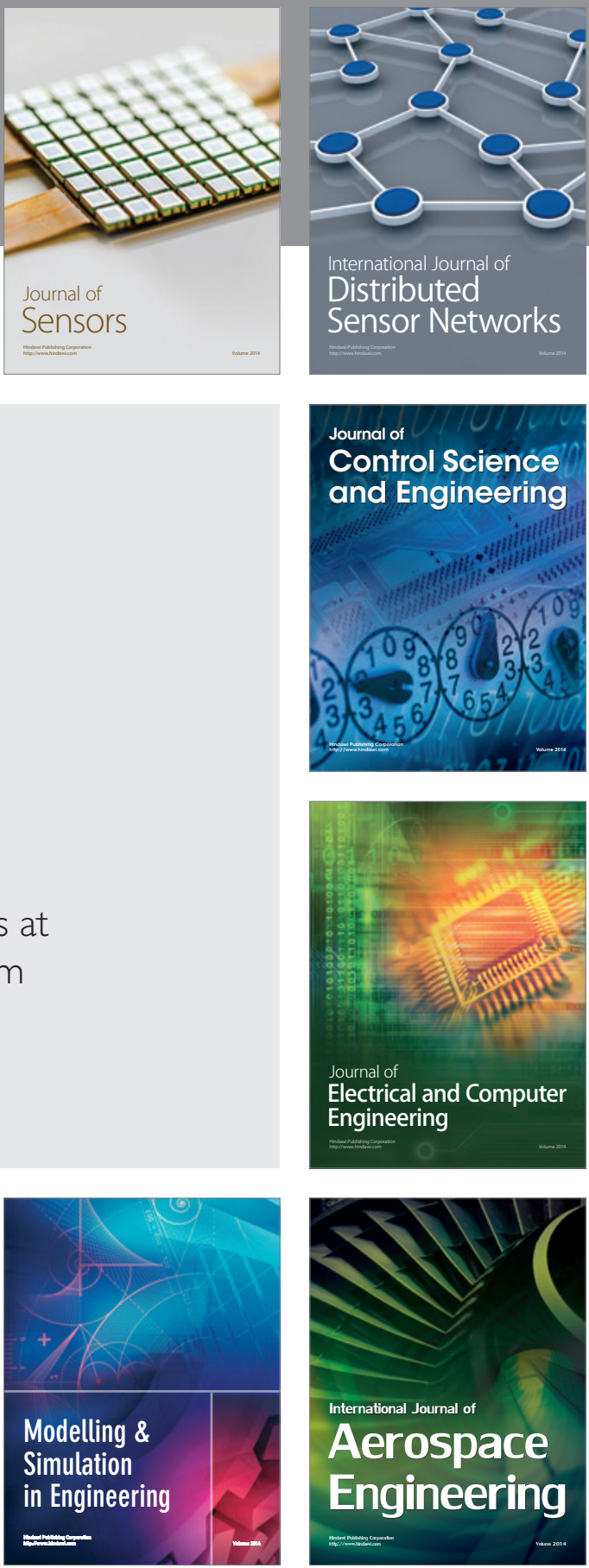

Journal of

Control Science

and Engineering
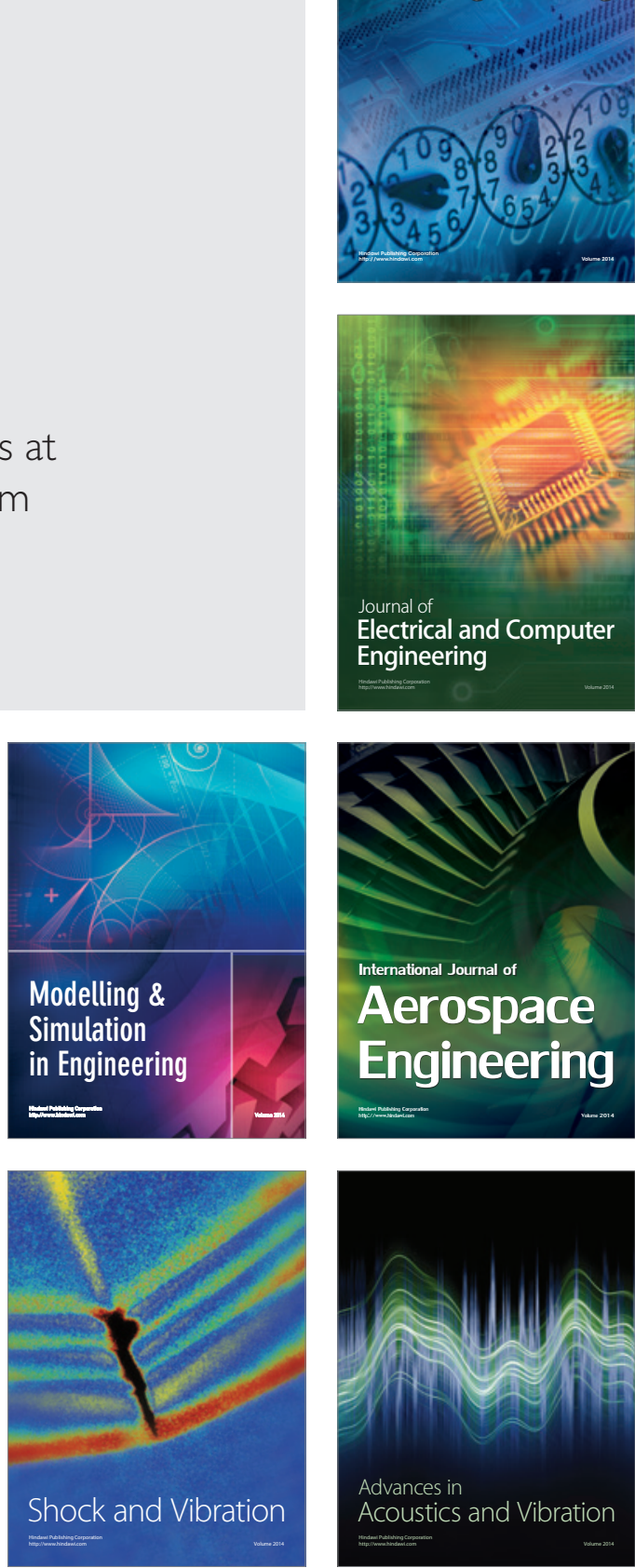